\title{
Incidencia de la gestión académica en la formación profesional de los estudiantes de la Maestría en Terapia Familiar Sistémica de la Universidad Politécnica Salesiana, período académico 2011-2012 The incidence of academic administration of student professional training in the Master's Family Systemic Therapy program at the Salesian Polytechnic University
}

\author{
Dorys Ortiz \\ Universidad Politécnica Salesiana \\ dortiz@ups.edu.ec
}

Recibido: 21 de febrero de 2013 / Aceptado: 28 de junio de 2013

\section{Resumen}

El objetivo de este texto es presentar los resultados de la investigación sobre la incidencia de la Gestión Académica en la Formación Profesional de los estudiantes de la Maestría en Terapia Familiar Sistémica de la Universidad Politécnica Salesiana. Se contextualiza este programa de formación en el conjunto de la universidad y se plantean los diversos problemas existentes en la gestión académica respecto a la docencia, investigación, gestión de recursos y vinculación con la sociedad y su relación con ciertos aspectos debilitados de la formación profesional como la admisión de los estudiantes y docentes, el desarrollo de líneas de investigación, el seguimiento a graduados y la difusión de resultados. Luego se define y se analizan teóricamente tanto la gestión académica como la formación profesional. En tercer lugar, se desarrollan los aspectos metodológicos de la investigación realizada que es de tipo correlacional. Los datos recopilados se presentan en tablas que permiten una rápida visualización de los resultados y contribuyen a verificar la incidencia de la gestión en la formación profesional al comprobar la hipótesis mediante la prueba Chi cuadrado. La información obtenida, finalmente, permite caracterizar la gestión académica y describir la formación profesional de la maestría.

\section{Palabras clave}

Gestión educativa, formación profesional, docencia, investigación, aprender a aprender, metacognición.

Forma sugerida de citar: Ortiz, D. (2013). Incidencia de la gestión académica en la formación profesional de los estudiantes de la Maestría en Terapia Familiar Sistémica de la Universidad Politécnica Salesiana, período académico 2011-2012. Alteridad, 8(1), pp. 81-96. Quito: Editorial Abya-Yala.

\begin{abstract}
The purpose of this paper is to present the results of research on the incidence of Academic Management in Vocational Training for students of the Master in Systemic Family Therapy in Salesian Polytechnic University. It contextualizes this training program in the entire university and raises various problems in academic management regarding the functions of teaching, research, resource management and links with society and its relation to certain aspects weakened vocational training, essentially, the admission of students and teachers, developing lines of research, monitoring graduates and the dissemination of results. Then, it defined and analyzed theoretically both academic management and vocational training. Third, it develops the methodological aspects of the research that is correlational. The collected data are presented in tables that allow quick visualization of results and help to check the influence of management on vocational training to test the hypothesis by Chi square test. The information obtained, finally, allows characterizing academic management and describing vocational training of the master.
\end{abstract}

\section{Key-words}

Academic management, vocational training, teaching, research, learning to learn, metacognition. 


\section{Introducción}

La Universidad Politécnica Salesiana es una institución de educación superior, cuya labor se orienta a la formación de pre y postgrado. $\mathrm{Su}$ labor se efectiviza gracias a las funciones de docencia, investigación, vinculación con la sociedad y gestión universitaria.

La Unidad de Postgrados está encargada de la formulación, ejecución y evaluación de programas de postgrado. Uno de ellos es la Maestría en Terapia Familiar Sistémica, durante cuya ejecución se han advertido dificultades en la gestión académica que inciden en la formación profesional de los estudiantes. En la docencia: ejecución de los módulos y evaluación de aprendizajes. En la investigación: dificultades en la formación. En la gestión de recursos: dificultades en la reglamentación interna, las funciones de la coordinación y las relaciones interpersonales. En la vinculación con la colectividad: problemas en el establecimiento de convenios interinstitucionales y la valoración del impacto.

El conjunto de dificultades señaladas, generan como consecuencia que el proceso formativo de los estudiantes se afecte, particularmente en el área de la metodología y la evaluación de los conocimientos, en el nivel de adquisición de estos, así como también en la integración de las habilidades y destrezas. Razón por la cual, investigar en esta área es de gran relevancia puesto que implica garantizar aspectos de calidad en la formación profesional.

La presente investigación ha planteado como hipótesis que la gestión académica incide en la formación profesional y se ha definido como objetivo establecer dicha incidencia, además de caracterizar los ámbitos de la gestión académica y describir la formación profesional.

\section{Marco Teórico}

Según Lucas Pacheco, la gestión académica: "se encarga de la dirección, coordinación y monitoreo de las actividades de docencia, investigación y extensión universitarias” (2008: 157).

Las acciones de dirección, coordinación y monitoreo de las actividades, se conocen como "funciones gerenciales", entre las que destacan la planificación. Camisón y Dalmau señalan: "Planificar... consiste en hacer planes; en definitiva, en idear una acción o conjunto de acciones futuras, e implica, en primer lugar, establecer o fijar los objetivos, $y$, posteriormente, diseñar las estrategias o cursos de acción necesarios para alcanzar esos objetivos" (2009: 199).

Los objetivos son esenciales para toda organización puesto que señalan y orientan las actividades que se van a realizar. El hecho de fijar un curso de acción, implica que la persona encargada de la gestión conoce los objetivos y además, sabe la forma de alcanzarlos.

Camisón y Dalmau señalan que la organización: "se refiere a la acción y efecto de organizar un conjunto de acciones que se llevan a cabo por la empresa para ordenar sus elementos, estableciendo las relaciones, dependencias e interacciones entre los distintos elementos, personas, tareas, recursos y responsabilidades" (2009: 199).

La organización proporciona la estructura formal que contribuye a que las personas implicadas en el proceso puedan realizar sus tareas de tal forma que el resultado sea el cumplimiento de los objetivos y el logro de las metas.

Camisón y Dalmau indican que la dirección "tiene por objeto influir en las actividades individuales y de grupo, orientándolas hacia el establecimiento y logro de las metas" (2009:200). Esta influencia pasa por características personales por lo que el gestor debe desarrollar las habilidades para orientar las actividades propias y ajenas de tal manera que no se sienta que se está obligado a hacerlo.

Los autores mencionados establecen que la fase de control y seguimiento: "permite cerrar o completar el proceso de administración al comprobar que los objetivos planteados efectivamente se logran" (2009: 200). Todos estos procesos se realizan gracias a los sistemas de seguimiento y 


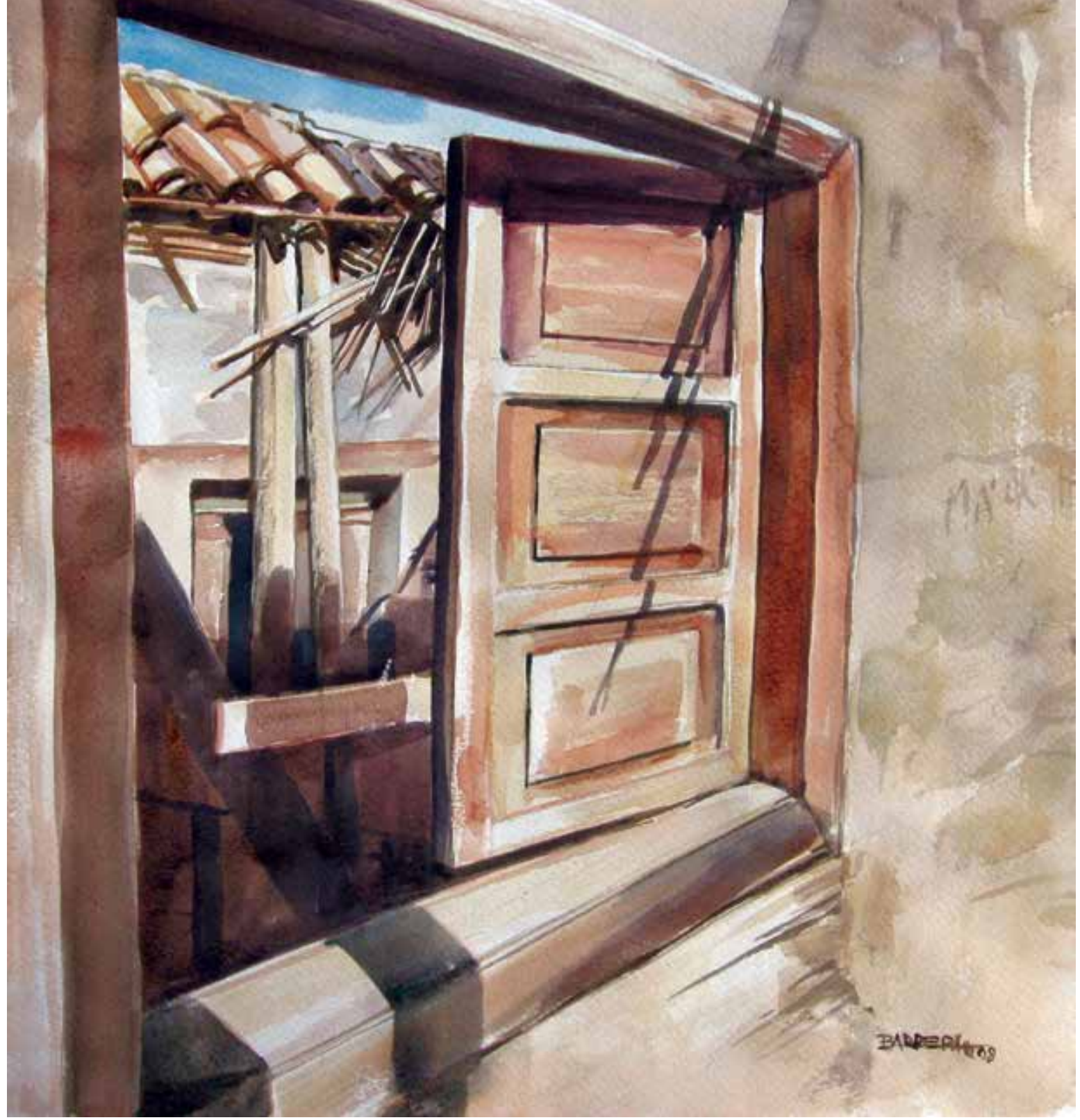

apoyo que son esenciales para facilitar la toma de decisiones en cualquier fase del proceso.

La gestión académica tiene que ver con varios ámbitos en el gran conjunto de actividades que se desarrollan en la universidad y que se detallan a continuación.

En el desarrollo curricular, la gestión académica debe tomar en cuenta:

a. Dirigir los estudios del macrocurrículo y programar las actividades del microcurrículo

b. Coordinar las tres clásicas actividades académicas: docencia, investigación y extensión c. La evaluación del currículo (Pacheco, 2008: 160).

En la investigación, según Pacheco la gestión académica debe preocuparse por: "conocer y manejar adecuadamente los arbitrios e instrumentos indispensables para dirigir la programación, seguimiento, evaluación, difusión, y si es el caso, la aplicación de los siguientes niveles de investigación: científica, tecnológica, para el desarrollo académico y cultura y para consultoría" (2008: 161). 
La calidad y excelencia, según lo señala el INNCREA, 2007 "Profundiza y enfatiza el cambio en el estilo de vida; es una filosofía dominante en donde los procesos de mejora continua y las decisiones en consenso prevalecen sobre el autoritarismo y el individualismo de la cultura tradicional [...]" (citado por N. Raza, 2008: 232).

La Ética, en el campo de la gestión académica, le corresponde interrogarse sobre los actos del gestor.

Además, la gestión y su desenvolvimiento se ven afectadas por muchos factores dentro y fuera de la institución que determinan el avance o estancamiento de la misma como la situación financiera y la interdependencia de los departamentos.

\section{Formación Profesional}

Existen muchas definiciones de "formación profesional", como la de Beau: "Formar adultos es conducir un grupo de adultos, que se denominará la población de inicio, de una

En la vinculación con la sociedad, la gestión académica se ocupa de: "el sistema educativo nacional, las relaciones con el Estado, las nacionalidades y culturas que conforman nuestro país y las relaciones académicas internacionales" (Pacheco, 2008: 160).

En la gestión del personal académico, Pacheco señala que: "se desenvuelve dentro de las políticas de selección, capacitación y evaluación del personal académico [...]" (2008: 162). Así el gestor también debe tener habilidades para tratar con las personas y poder establecer criterios adecuados para su implicación en el proceso.

Existen muchos principios que orientan la gestión académica, se enfatiza tres de ellos:

La transparencia, constituye un principio básico de la gestión académica. Según Ruth Mesta significa: “[...] el deber de las Autoridades de realizar sus acciones de manera pública, como un mecanismo de control del poder y de legitimidad democrática de las instituciones públicas" (2006: 1). situación inicial (que se tratará de caracterizar) a una situación final (que llamaremos el objetivo de la formación) por la puesta en marcha de medios, que llamaremos el sistema pedagógico (2000: 21).

Este proceso requiere la formulación de un modelo educativo, que según Lamata y Domínguez: "es la explicación de por qué queremos hacer formación, en base a qué planteamientos educativos y de aprendizaje la fundamentamos y cómo desarrollamos los distintos elementos que componen el proceso formativo" (2003: 19).

La construcción del proceso formativo es una compleja red cuyo primer paso, según Lamata y Domínguez (2003) es determinar cuáles son las necesidades que una población dada, tiene en un ámbito específico, luego hay que definir los objetivos de la formación, que constituyen la guía del proceso de formación, determinan el orden de los contenidos y su secuencia, orientan los métodos, definen la evaluación y guardan una estrecha relación con las estrategias de aprendizaje. 
Toda formación requiere de unos contenidos que están formados por toda la información que se propone y/o genera en el proceso formativo. Estos contenidos se dan con base a una metodología que se traduce en técnicas, que según Lamata y Domínguez, se pueden definir como: "instrumentos, herramientas que se aplican durante el proceso formativo" (2003: 197).

Es esencial evaluar el proceso formativo para obtener información sobre la ejecución del mismo y el cumplimiento de los objetivos. Existe una amplia discusión acerca del por qué y para qué se evalúa, en la que se plantean diversas posturas, sin embargo, la mayoría de ellas, coinciden en que la evaluación, según Rosales se realiza para: recoger información sobre los componentes y actividades de la enseñanza, interpretar esta información de acuerdo con una determinada teoría o esquema conceptual y adoptar decisiones relativas al perfeccionamiento del sistema (2000: 32).

\section{Metodología}

En la investigación se utilizaron el método científico y el hipotético-deductivo. Como técnica se usó la encuesta y se construyó un cuestionario, cuya validez fue comprobada con un procedimiento interjueces y la confiabilidad, mediante una prueba piloto cuyos resultados fueron evaluados, mediante el coeficiente Alpha de Cronbach, obteniendo un nivel de 87,6\%.

El instrumento cuenta con cinco preguntas demográficas y dieciséis preguntas cerradas correspondientes a los indicadores establecidos en las variables de investigación: gestión académica y formación profesional.

La población está conformada por 54 estudiantes, la muestra es equivalente a la población debido a que es limitada y se escogió de manera no probabilística e intencional.

El instrumento se aplicó en condiciones adecuadas de tiempo y de disponibilidad de los participantes. Una vez recogida toda la informa- ción, se procedió a su procesamiento, con una revisión crítica de los datos recopilados para eliminar aquellos que tuviesen información defectuosa, con lo cual se eliminaron tres encuestas debido a que no fueron respondidas en su totalidad. Luego, se tabularon los datos en tablas de contingencia, se realizó el análisis estadístico mediante pruebas descriptivas, se realizó una interpretación de los resultados, y, finalmente, se utilizó el programa SPSS versión 18 y se procedió a verificar la hipótesis.

\section{Análisis y resultados}

En la encuesta se plantearon varias preguntas sobre datos demográficos, cuyos resultados se sintetizan a continuación.

El $23.53 \%$ de la población de estudiantes se encuentra entre los 45 y 49 años de edad, seguido por las personas entre 40 y 44 años, con un porcentaje del $19.61 \%$. También se puede identificar personas entre los 30 y 34 años de edad, con un $15.69 \%$. La gran diversidad de edades favorece el intercambio de experiencias entre personas que se encuentran en momentos diferentes de su carrera profesional: ya sea comenzando o casi al terminar.

Existe una primacía femenina, con el $78.43 \%$, aspecto que confirma la vocación femenina por los roles de ayuda y de acompañamiento a otras personas. En cuanto a la profesión: el gran porcentaje $(56.85 \%)$ corresponde a psicólogos/as (clínicos, educativos, organizacionales). Es posible pensar que la maestría fortalece las capacidades y habilidades de este segmento profesional y les brinda mejores herramientas para su desempeño profesional.

Los estudiantes encuestados pertenecen a cuatro promociones de la maestría. Existe una distribución similar en cada promoción. Se observa sin embargo, una ligera primacía de las personas de la segunda promoción, con un $31.37 \%$, seguidas por las de las primera con un $25.49 \%$. 


\section{Admisión de estudiantes y la selección de docentes}

El 78.43\% de los estudiantes está de acuerdo en que ambos aspectos tienen una planificación adecuada. Esto revela que el procedimiento por el cual, ellos fueron admitidos a la maestría estuvo claramente planteado. Sin embargo, hay que considerar el leve porcentaje de $21.57 \%$ de los estudiantes que manifestaron un acuerdo parcial, ya que quizá el procedimiento no estuvo lo suficientemente explícito para ellos.

\section{Tabla 1. Admisión de estudiantes y selección docentes}



\begin{tabular}{|l|l|c|c|}
\hline \multirow{3}{*}{1} & De acuerdo & 40 & 78,43 \\
\hline \multirow{1}{*}{$\begin{array}{l}\text { Parcialmente de } \\
\text { acuerdo }\end{array}$} & 11 & 21,57 \\
\hline & En desacuerdo & 0 & 0,00 \\
\hline & Total & 51 & 100,00 \\
\hline
\end{tabular}

Con respecto a la admisión y selección de docentes, los estudiantes están de acuerdo en que existía un procedimiento para hacerlo, sin embargo, manifestaban verbalmente que no conocían cómo se hacía esta selección.

\section{Existencia de una planificación anual para la ejecución de los módulos}

Los estudiantes están plenamente de acuerdo con esta afirmación, alcanzando un porcentaje del $96.08 \%$; es decir, 9 de cada 10 estudiantes consideran que la ejecución de los módulos se realizaba gracias a una planificación anual.

\section{Tabla 2. Planificación y ejecución de módulos}

\begin{tabular}{|c|c|c|c|}
\hline 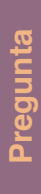 & 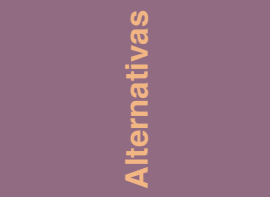 & $\begin{array}{l}\frac{\pi}{0} \\
\frac{\mathrm{C}}{6} \\
\frac{\mathrm{d}}{\mathrm{d}} \\
\frac{\mathrm{L}}{4}\end{array}$ & 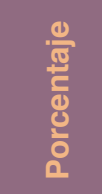 \\
\hline \multirow{4}{*}{2} & De acuerdo & 49 & 96,08 \\
\hline & $\begin{array}{l}\text { Parcialmente de } \\
\text { acuerdo }\end{array}$ & 2 & 3,92 \\
\hline & En desacuerdo & 0 & 0,00 \\
\hline & Total & 51 & 100,00 \\
\hline
\end{tabular}

\section{Definición clara de los parámetros para evaluar los aprendizajes}

Las respuestas de los estudiantes a esta pregunta, señalan que un $74.51 \%$ de ellos están de acuerdo y solo un $25.49 \%$ están parcialmente de acuerdo, con lo cual, toda la población, de una u otra forma ha conocido que la evaluación tiene ciertos criterios y responde a parámetros fijados.

Tabla 3. Evaluación de aprendizajes

\begin{tabular}{|c|c|c|c|}
\hline 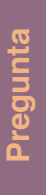 & 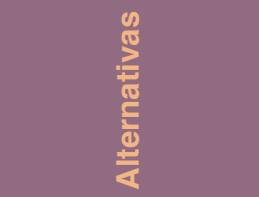 & 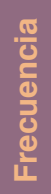 & 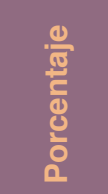 \\
\hline \multirow{4}{*}{3} & De acuerdo & 38 & 74,51 \\
\hline & $\begin{array}{l}\text { Parcialmente de } \\
\text { acuerdo }\end{array}$ & 13 & 25,49 \\
\hline & En desacuerdo & 0 & 0,00 \\
\hline & Total & 51 & 100,00 \\
\hline
\end{tabular}

Los estudiantes responden concretamente que los aspectos de la docencia influyen directamente sobre el proceso, con un $68.63 \%$. La 
docencia es el núcleo de la formación, a ella están vinculados diversos niveles: coordinación, docentes, estudiantes y el proceso mismo, razón por la cual, el influjo se ve netamente en este.

Tabla 4. Influencia de la Docencia en la Formación Profesional

\begin{tabular}{|c|c|c|c|c|}
\hline 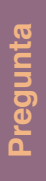 & $\begin{array}{l}\text { 뜬 } \\
\text { 용 } \\
\text { 읶 }\end{array}$ & 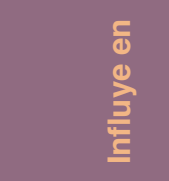 & 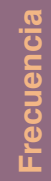 & $\begin{array}{l}\frac{0}{30} \\
\frac{3}{0} \\
\frac{d}{0} \\
\frac{0}{0} \\
2\end{array}$ \\
\hline \multirow{4}{*}{4} & \multirow{4}{*}{$\begin{array}{l}\frac{\pi}{0} \\
\frac{\pi}{0} \\
0 \\
0 \\
0\end{array}$} & Proceso & 35 & 68,63 \\
\hline & & Personas & 3 & 5,88 \\
\hline & & Resultados & 13 & 25,49 \\
\hline & & Total & 51 & 100,00 \\
\hline
\end{tabular}

\section{Formación en investigación}

Las respuestas a esta pregunta están bastante divididas, puesto que el $49.02 \%$ de los estudiantes está de acuerdo con la afirmación, pero existe un alto porcentaje que están parcialmente de acuerdo (el $39.22 \%$ ) y 6 personas (11.76\%) manifiestan su desacuerdo. Esto da a notar que la formación en investigación no es un eje todavía bien definido, ya que genera respuestas tan variadas.

\section{Tabla 5. Formación en investigación}

\begin{tabular}{|c|c|c|c|}
\hline 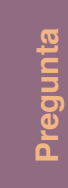 & 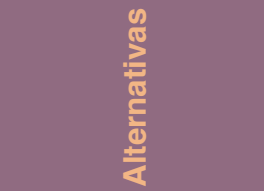 & 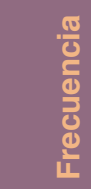 &  \\
\hline \multirow{4}{*}{5} & De acuerdo & 25 & 49,02 \\
\hline & $\begin{array}{l}\text { Parcialmente de } \\
\text { acuerdo }\end{array}$ & 20 & 39,22 \\
\hline & En desacuerdo & 6 & 11,76 \\
\hline & Total & 51 & 100,00 \\
\hline
\end{tabular}

Los estudiantes manifiestan que la investigación influye directamente sobre los resultados obtenidos, lo cual es coherente con el análisis efectuado anteriormente.

Tabla 6. Influencia de la Investigación sobre la Formación Profesional

\begin{tabular}{|c|c|c|c|c|}
\hline 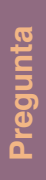 &  & 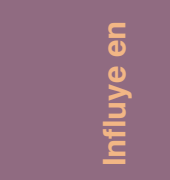 & 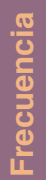 & 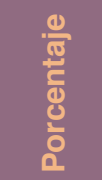 \\
\hline \multirow{4}{*}{4} & \multirow{4}{*}{ 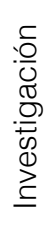 } & Proceso & 18 & 35,29 \\
\hline & & Personas & 9 & 17,65 \\
\hline & & Resultados & 24 & 47,06 \\
\hline & & Total & 51 & 100,00 \\
\hline
\end{tabular}

\section{Existencia de un instructivo}

Existe una amplia división en las respuestas aportadas por los estudiantes. Mientras el $49.02 \%$ está de acuerdo en la existencia de un instructivo, un $43.14 \%$ demuestra un acuerdo parcial con el tema y un $7.84 \%$ está en franco desacuerdo.

\section{Tabla 7. Existencia de instructivos (estudiantes)}

\begin{tabular}{|c|c|c|c|}
\hline$\frac{\text { 를 }}{8}$ & $\frac{0}{\frac{0}{30}}$ & $\frac{\text { du }}{\frac{0}{6}}$ & $\begin{array}{l}\frac{0}{8} \\
\frac{\mathrm{e}}{0} \\
\frac{8}{2} \\
\frac{0}{0}\end{array}$ \\
\hline \multirow{4}{*}{7} & De acuerdo & 25 & 49,02 \\
\hline & $\begin{array}{l}\text { Parcialmente de } \\
\text { acuerdo }\end{array}$ & 22 & 43,14 \\
\hline & En desacuerdo & 4 & 7,84 \\
\hline & Total & 51 & 100,00 \\
\hline
\end{tabular}

La tabla anterior revela posiblemente un cierto desconocimiento sobre la inclusión de ciertos aspectos en los instructivos, ya que si bien es cierto, se proporcionaba un instructivo de tesis a los estudiantes, no se les proporcionaba un ins- 
tructivo de la maestría, razón por la cual desconocían las funciones de la dirección.

\section{Disponibilidad de recursos de todo tipo para llevar a cabo la formación}

Los estudiantes manifiestan su acuerdo con la existencia de recursos para la formación, en un $50.98 \%$. Sin embargo existe un alto porcentaje, $47.06 \%$ que están parcialmente de acuerdo con esta idea, quizá porque hacen énfasis en el tema de la existencia de "todo tipo de recursos", que, históricamente, para las personas de la primera promoción no estuvieron tan disponibles como para los de la cuarta.

Tabla 8. Disponibilidad de recursos



Los estudiantes, en un porcentaje de 49.02\% afirman que los aspectos de la gestión de recursos influyen directamente sobre el proceso de formación. Esto va de acuerdo con la realidad encontrada, ya que las relaciones son esenciales para el logro de los objetivos, pero también influye sobre esto, la disponibilidad de recursos que proporcionan una base sobre la cual realizar las actividades.

\section{Tabla 9. Influencia de la Gestión de Recursos en la Formación Profesional}

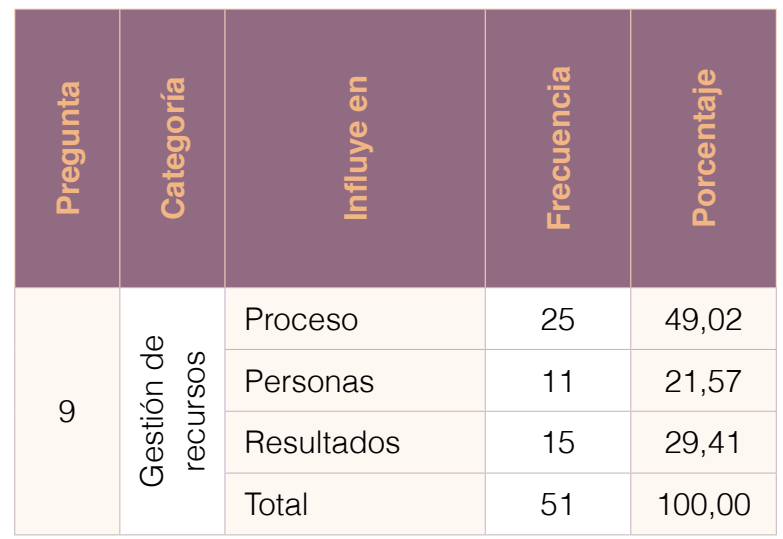

\section{Vínculos con organizaciones nacionales e internacionales}

Existe un alto grado de acuerdo sobre vínculos con otras organizaciones. El $64.71 \%$ de los estudiantes está de acuerdo con esta afirmación, mientras solo un $31.37 \%$ está parcialmente de acuerdo, quizá debido a que desconocían sobre el tema o no se han involucrado en actividades relacionadas con el trabajo con la sociedad.

\section{Tabla 10. Vínculos con organizaciones}

\begin{tabular}{|c|c|c|c|}
\hline 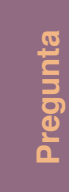 & &  & 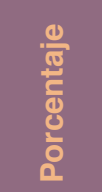 \\
\hline \multirow{4}{*}{10} & De acuerdo & 33 & 64,71 \\
\hline & $\begin{array}{l}\text { Parcialmente de } \\
\text { acuerdo }\end{array}$ & 16 & 31,37 \\
\hline & En desacuerdo & 2 & 3,92 \\
\hline & Total & 51 & 100,00 \\
\hline
\end{tabular}


Los estudiantes manifiestan que los aspectos de la vinculación se relacionan específicamente con los resultados obtenidos en el proceso de formación, afirmación que alcanza un $56.86 \%$. Sin embargo, también hay opiniones que éste influye sobre las personas, con un $23.53 \%$ y el proceso, con un $19,61 \%$.

Tabla 11 . Influencia de la Vinculación con la Sociedad en la Formación Profesional



\section{El proceso formativo estableció una demanda de formación, unos objetivos y unos contenidos}

Los estudiantes, en un porcentaje que alcanza el $86.27 \%$ están de acuerdo en que la formación planteó una demanda, unos objetivos y unos contenidos. Existe un $13.73 \%$ de estudiantes que están parcialmente de acuerdo con esta afirmación, probablemente porque carecen de información al respecto.
Tabla 12. Demanda de formación, objetivos y contenidos de la formación

\begin{tabular}{|c|c|c|c|}
\hline 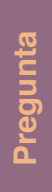 & 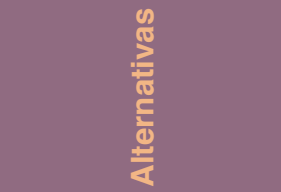 & 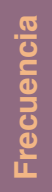 &  \\
\hline \multirow{4}{*}{12} & De acuerdo & 44 & 86,27 \\
\hline & $\begin{array}{l}\text { Parcialmente de } \\
\text { acuerdo }\end{array}$ & 7 & 13,73 \\
\hline & En desacuerdo & 0 & 0,00 \\
\hline & Total & 51 & 100,00 \\
\hline
\end{tabular}

\section{La metodología, técnicas y herramien- tas permiten alcanzar los objetivos pro- puestos}

Los estudiantes manifiestan estar de acuerdo con esta pregunta, en un $84.31 \%$, lo cual es un porcentaje elevado. Existe apenas un $15.69 \%$ que está parcialmente de acuerdo con el tema, tal como lo muestra la tabla y la figura correspondiente.

\section{Tabla 13. Metodología, técnicas y herramientas}






\section{La evaluación de la formación ofrece retroalimentación sobre el proceso for- mativo}

Los estudiantes están de acuerdo con estas pregunta, en un $80.39 \%$ con lo cual se puede afirmar que la evaluación dada y recibida favorece la retroalimentación sobre el proceso. Cabe destacar sin embargo que existe un porcentaje de estudiantes, el $17.65 \%$ que están parcialmente de acuerdo con esta afirmación, con lo cual tal vez es importante reconsiderar el tema de la evaluación para mejorarla.

Tabla 14. Evaluación de la formación

\begin{tabular}{|c|c|c|c|}
\hline 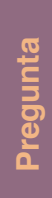 & $\frac{\text { d }}{\frac{\mathrm{d}}{\mathrm{d}}}$ &  & 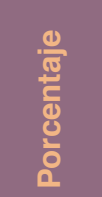 \\
\hline \multirow{4}{*}{14} & De acuerdo & 41 & 80,39 \\
\hline & $\begin{array}{l}\text { Parcialmente de } \\
\text { acuerdo }\end{array}$ & 9 & 17,65 \\
\hline & En desacuerdo & 1 & 1,96 \\
\hline & Total & 51 & 100,00 \\
\hline
\end{tabular}

\section{La administración de la universidad contribuye a la realización de las activi- dades de la formación}

Los estudiantes demuestran una posición un tanto dividida respecto a este tema. El 54.9\% está de acuerdo en que la administración contribuye a la realización de las actividades, mientras que un $43.14 \%$ señala que está parcialmente de acuerdo con esta afirmación, lo cual puede revelar un cierto grado de inconformidad o insa- tisfacción en las acciones llevadas a cabo por los administrativos.

Tabla 15. Agentes de la formación

\begin{tabular}{|c|c|c|c|}
\hline 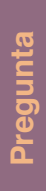 & 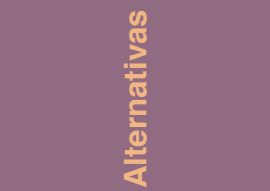 & 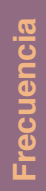 & $\begin{array}{l}\frac{0}{8} \\
\frac{8}{0} \\
\frac{0}{0} \\
\frac{0}{0} \\
\text { L }\end{array}$ \\
\hline \multirow{4}{*}{15} & De acuerdo & 28 & 54,90 \\
\hline & $\begin{array}{l}\text { Parcialmente de } \\
\text { acuerdo }\end{array}$ & 22 & 43,14 \\
\hline & En desacuerdo & 1 & 1,96 \\
\hline & Total & 51 & 100,00 \\
\hline
\end{tabular}

\section{Impacto de la maestría}

El 80.39\% de los estudiantes están de acuerdo con esta afirmación, lo que revela que la maestría tiene un impacto que incluso se puede medir, en función del número de graduados. Existe un $17.65 \%$ que está parcialmente de acuerdo con esta afirmación, lo cual puede revelar la falta de difusión de los resultados obtenidos por los docentes en otras actividades y que no se comentaban a los estudiantes.

\section{Tabla 16. Impacto}

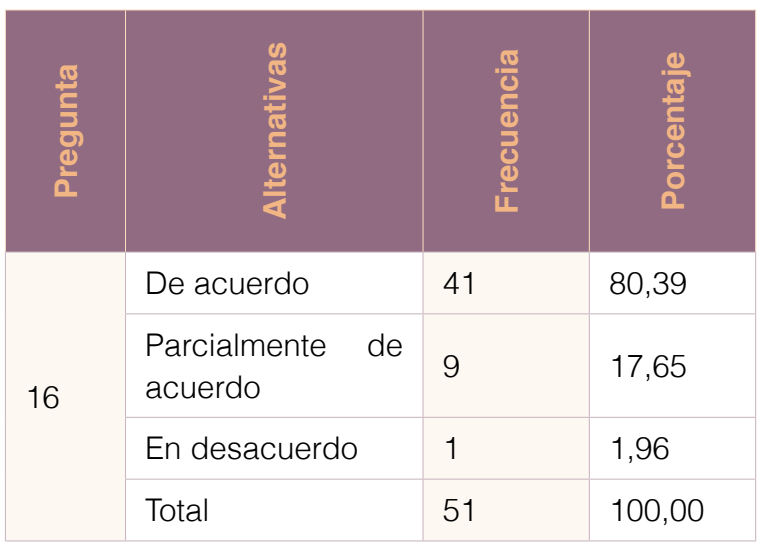




\section{Discusión y conclusiones}

Según los resultados lo muestran, existe coincidencia con Lucas Pacheco, quien señala que la gestión académica: "se encarga de la dirección, coordinación y monitoreo de las actividades de docencia, investigación y extensión universitarias" (2008: 157).

Tal como se ha podido observar en los resultados, la planificación está bastante clara para los estudiantes. La teoría señala que este momento implica dos aspectos esenciales: fijar los objetivos y fijar el curso de acción. Los resultados señalan claramente que los estudiantes perciben estos procesos llevados a cabo para planificar las actividades de la maestría.

Se puede decir entonces, que la gestión académica se basa en una planificación previa que los estudiantes, la reciben como destinatarios de ella, pero es posible que no sean muy conscientes de todo el trabajo que existe detrás. En cuanto a la fijación del curso de acción, es posible darse cuenta del énfasis dado al proceso en cada uno de los ítems abordados. Existe una clara manifestación de este curso de acción en las actividades de la maestría, que es claramente percibido.

Mientras que la planificación fija la orientación y la forma de alcanzar la meta propuesta, la organización proporciona la estructura necesaria para que lo planificado se ejecute. Los resultados muestran que la maestría tiene un nivel bueno de organización, puesto que cada persona sabe cuáles son sus tareas y su contribución a los objetivos definidos.

Un componente esencial de la organización, en el caso de la MIATEFAS, ha sido la "conformación de un equipo de trabajo", algunas de sus ventajas son: la diversidad de los puntos de vista, en función del género (hombres y mujeres), la edad (adultos jóvenes y adultos en la tercera edad), la profesión (médicos, psicólogos, sociólogos), el recorrido profesional (institucional, personal) y el recorrido profesional (profesionales “junior" y otros "senior").
Esta diversidad -y más aún- la integración de esta diversidad en una visión más o menos consensuada, ha contribuido a un enriquecimiento, aprendizaje y crecimiento personal y profesional.

En el área de la gestión académica, la teoría señala la importancia de considerar algunos principios básicos y en el caso del presente trabajo, se escogieron fundamentalmente: la transparencia, la calidad y la ética. Entre los tres principios el más señalado es el de la ética. En cambio, el tema de la transparencia no es abordado específicamente, sin embargo, se puede percibir implícitamente, puesto que los resultados hacen referencia a diversos aspectos como la planificación, la formación en investigación, el impacto de la maestría. Este nivel de información poseído, implica necesariamente cierto grado de transparencia en la gestión, caso contrario, no podrían señalar estos aspectos.

De igual manera, los estudiantes perciben la planificación, en el planteamiento de la evaluación de los aprendizajes, puesto que están de acuerdo con la pregunta realizada. La evaluación es un momento clave de la planificación puesto que ofrecerá retroalimentación valiosa sobre el proceso (Rosales, 2000).

Estos aspectos vinculados a la docencia, remiten a pensar que ésta área influye mucho sobre la formación profesional puesto que es la más cercana a los estudiantes y que los implica bastante puesto que se están formando.

Según Pacheco (2008), la gestión académica en la investigación se interesa básicamente por la planificación, ejecución, seguimiento, evaluación y difusión de las actividades de investigación.

Respecto a este tema, los resultados de la investigación señalan que el proceso comenzó en el segundo año de trabajo de la maestría y que sufrió un cambio evolutivo.

Según Pacheco (2008), la gestión de recursos hace referencia esencialmente al personal académico. Sin embargo, también ha sido posible darse cuenta, que al hablar de "recursos" se remite a todo elemento que ayuda al desarrollo de la formación y en consecuencia tiene que ser considerado y gestionado: esto va desde la infraestructura 
(aulas), hasta los materiales y equipos para las clases y los refrigerios ofrecidos durante las jornadas de formación.

La maestría ha realizado trabajos de investigación como muchas otras organizaciones e instituciones a nivel nacional e internacional. También se destaca en la gestión académica de la maestría la inclusión en una red internacional, denominada RELATES, que tuvo como consecuencias favorables la formación docente y el posicionamiento de la maestría a nivel nacional.

\section{Descripción de la formación profesional}

Los resultados de la investigación señalan que la formación profesional en la maestría se hizo mediante la planificación adecuada (lo cual tiene que ver con la gestión también). Esta planificación contó con el establecimiento de una demanda, unos objetivos, los contenidos, la metodología, las técnicas y la evaluación (Lamata y Domínguez, 2003).

Además, cabe señalar que la formación profesional se hace con base al modelo educativo (Lamata y Domínguez, 2003), denominado como generativo. Antonio Ontoria (2003) sostiene que el conocimiento no se refiere a información, ya que mientras esta se compone de datos y hechos, aquel se refiere al hecho de comprender y darle un significado a la información obtenida.

El aprendizaje que se realiza durante la formación sistémica se plantea como un "proceso". Hunt (1997, citado por Ontoria 2003: 56) señala la distinción entre el aprendizaje como proceso y al aprendizaje como producto:

El proceso es el "cómo" del aprendizaje; es la manera que tenemos de aprender. El producto es el "qué" del aprendizaje; es la materia, asignatura o programa con que estamos trabajando $[. . .$.$] Los beneficios conseguidos en el$ proceso suelen ser tan valiosos para el objetivo de aprender durante toda la vida como el producto (Ontoria, 2003: 74).
A este tipo de aprendizaje se le ha dado el nombre de "aprendizaje generativo" o de segundo orden ya que tiende a transformar los modelos mentales, los cuales son referentes para la acción.

En este proceso, se espera que las personas utilicen estrategias cognitivas para el procesamiento de la información, fundamentalmente, la metacognición. Fue Flavell (1971) quien acuñó este término. Existen muchas formas de definirlo:

Manera de aprender a razonar sobre el propio razonamiento, aplicación del pensamiento al acto de pensar, aprender a aprender, es mejorar las actividades y las tareas intelectuales que uno lleva a cabo usando la reflexión para orientarlas y asegurarse una buena ejecución. (Yael Abramovicz Rosenblatt, 2012).

Metacognición es un término que se usa para designar a una serie de operaciones, actividades y funciones cognoscitivas llevadas a cabo por una persona, mediante un conjunto interiorizado de mecanismos intelectuales que le permiten recabar, producir y evaluar información, a la vez que hacen posible que dicha persona pueda conocer (P. Zenteno).

En el caso de la maestría, este aprendizaje se da en cuatro ejes:

El saber: Está constituido por la epistemología sistémica que sustenta la formación y que se estudia en tres niveles: bases, modelos de terapia familiar sistémica y aplicaciones.

El hacer: hace referencia a las habilidades y destrezas que un terapeuta familiar sistémico adquiere y que contribuyen a que pueda ayudar a otras personas en dificultades.

El ser: Constituye el eje en el que se realiza una revisión del profesional durante todo su proceso de formación.

La investigación: Este eje se refiere esencialmente a la formación en investigación y que, en definitiva, contribuyen a la realización de la tesis.

Gracias a estos elementos, se puede afirmar que la maestría tiene un impacto personal en los maestrantes, pero también un impacto en la 
sociedad gracias a los resultados obtenidos como sus investigaciones, publicaciones, etc.

Finalmente, con la información obtenida acerca de la influencia de la gestión sobre la for- mación profesional se puede verificar la hipótesis planteada, mediante la prueba de Chi cuadrado.

Tabla 17. Tabla de contingencia Gestión académica vs. Formación profesional

\begin{tabular}{|c|c|c|c|c|c|}
\hline & & \multicolumn{3}{|c|}{ Formación profesional } & \multirow[b]{2}{*}{ Total } \\
\hline & & Proceso & Personas & Resultados & \\
\hline \multirow{5}{*}{$\begin{array}{l}\text { Gestión } \\
\text { Académica }\end{array}$} & Docencia & 35 & 3 & 13 & 51 \\
\hline & Investigación & 18 & 9 & 24 & 51 \\
\hline & Gestión & 25 & 11 & 15 & 51 \\
\hline & Vinculación & 10 & 12 & 29 & 51 \\
\hline & Total & 88 & 35 & 81 & 204 \\
\hline
\end{tabular}

Tabla 18. Prueba de chi-cuadrado

\begin{tabular}{|c|c|c|c|}
\hline & 흘 & $\overline{5}$ &  \\
\hline $\begin{array}{l}\text { Chi-cuadrado de } \\
\text { Pearson }\end{array}$ & $29,367 a$ & 6 & 0 \\
\hline $\begin{array}{l}\text { Razón de verosi- } \\
\text { militudes }\end{array}$ & 31,058 & 6 & 0 \\
\hline $\begin{array}{l}\text { Asociación lineal } \\
\text { por lineal }\end{array}$ & 13,502 & 1 & 0 \\
\hline $\begin{array}{l}\text { N de casos váli- } \\
\text { dos }\end{array}$ & 204 & & \\
\hline \multicolumn{4}{|c|}{$\begin{array}{l}\text { a. } 0 \text { casillas }(0 \%) \text { tienen una frecuencia esperada } \\
\text { inferior a } 5 . \text { La frecuencia mínima esperada es } \\
8,75 \text {. }\end{array}$} \\
\hline
\end{tabular}

Como la significación es igual a 0,000 menor que 0,05 se puede afirmar con un $95 \%$ de probabilidad que la Gestión Académica incide en la Formación Profesional. Con lo cual, quedaría demostrada la hipótesis y se habría cumplido el objetivo general de la investigación planteada.

\section{Conclusiones}

En la contextualización del trabajo se abordó la descripción de la Universidad Politécnica Salesiana, la Unidad de Estudios de Postgrado y la maestría, cual contribuyó a fortalecer la ubicación de la investigación en un marco físico concreto que delimita ciertos aspectos esenciales para el proceso.

Los aspectos abordados en la teoría sobre la Gestión Académica proporcionaron una base importante para comprenderla de mejor manera. Sin duda alguna, la profundización y la mejor comprensión de los ámbitos y de los diversos aspectos de la gestión académica fortalecerán el trabajo que se realice en el futuro. Lo anterior es un gran resultado, bastante inesperado además, del proceso de investigación.

En el tema de la Formación Profesional fue importante realizar la revisión teórica para reflexionar sobre ciertos aspectos que estaban implícitos en el proceso y que han salido a la luz gracias a la lectura de los textos y la construcción del marco teórico. Uno de ellos es el reconocimiento del modelo de aprendizaje en la maestría, que se ha caracterizado como "generativo". 
También fue importante revisar la construcción del proceso formativo y darse cuenta de la interrelación existente entre los diversos niveles implicados: desde el análisis de las necesidades de formación, la definición de objetivos, los contenidos, los métodos, las técnicas, los recursos y la evaluación. Se ha podido establecer claramente la importancia de estos niveles y sus condicionantes, así como también su mutua influencia y la necesidad de definir criterios que contribuyan a su evaluación para ir mejorando el proceso.

En el momento de la ejecución de la investigación, el mayor desafío fue el de la construcción y validación de los instrumentos de investigación. Encontrar las preguntas adecuadas, formularlas de la mejor manera posible considerando los destinatarios y validar los instrumentos, fue una tarea complicada. Sin embargo, la revisión de todos estos aspectos y el cuidado en lograr buenos instrumentos de investigación dio sus frutos posteriormente, durante el procesamiento de la información que, en cambio, se facilitó enormemente debido a la posibilidad de pasar la información a los archivos en formato digital.

Durante la ejecución de la investigación, la recolección de información constituyó un reto debido a que había que localizar a los estudiantes de la maestría uno por uno para aplicar la encuesta. Sin embargo, la ayuda de los miembros del equipo de docentes fue inestimable para alcanzar a realizar el trabajo. Esta parte del proceso, tuvo un resultado inesperado puesto que fue enriquecedor para todos los participantes, incluida la investigadora, volver a tomar contacto con los estudiantes y ser testigos de sus recorridos profesionales, de las puertas que se les han abierto y del desarrollo que han tenido luego de haber finalizado la formación.

Los resultados obtenidos en la investigación contribuyeron al logro de los objetivos definidos en el plan, tanto de los específicos como del general. Así, con la información obtenida se pudo caracterizar la gestión académica de la maestría, describir su proceso de formación bajo parámetros de calidad y, lo más importante, demostrar la incidencia de la gestión académica en la formación profesional, hipótesis que tiene un $95 \%$ de probabilidad a favor.

A modo de conclusión final, se puede afirmar con énfasis que el proceso realizado ha contribuido a un aprendizaje decisivo respecto a dos temas esenciales para toda persona vinculada a una institución de educación y máxime si se trata de una de tipo universitario. Estas dos áreas son la gestión académica y la formación profesional: el conocimiento se ha enriquecido con aspectos técnicos, pero también con el reconocimiento del camino recorrido que ha contribuido, indudablemente, de manera definitiva a la construcción de un proceso vital para todos sus participantes. Esto renueva la fe y la esperanza.... el camino no ha sido en vano....

\section{Bibliografía}

Ali Zwain, A., Kong Teong, L. \& Norezam Othman, S. 2012. Knowledge Management Processes and Academic Performance in Iraqi HEIs: An Empirical Investigation. International Journal of Academic Research in Business and Social Sciences. Recuperado de: http://go.galegroup. com/ps/retrieve.do

Apodaca, P. y Lobato, C. 1997. Calidad en la Universidad: Orientación y Evaluación. Barcelona: Editorial Laertes.

Balanza, S., Morales, I., Guerrero, J. y Conesa, A. 2008. "Fiabilidad y validez de un cuestionario para medir en estudiantes universitarios la asociación de la ansiedad y depresión con factores académicos y psicosocio-familiares durante el curso 2004-2005". Revista Española de Salud Pública. Vol. 82. No. 2.

Beau, D.

2000. La boite à outils du formateur. 100 fiches pédagogiques. 3ième édition. Paris: Éditions d'Organisation, citado por: LIMOGES, Isabelle y MIVILLE DE-CHENE, Karl, $(\mathrm{s} / \mathrm{r})$. Formation de formateurs. Centre de Formation en Commerce International.

Camisón, C. y Dalmau, J. (Coord.).

2009. Introducción a los negocios y su gestión. España: Pearson Educación S.A. 
Castillo, S.

2006. Formación del profesorado en Educación Superior. Desarrollo curricular y evaluación. Vol. II. Madrid: McGraw-Hill.

Cuatrecasas, L.

2005. Gestión integral de la calidad. Implantación, control y certificación. España: Ediciones Gestión.

Dimas, G.Goula, a. \&Pierrakos, G.

2011. Quality Issues in Higher Education: A Multicriteria Framework of Satisfaction Measures. Atenas: Technological Education Institute of Athens en Creative Education. Vol. 2. No. 3.. 305-312. SciRes.

Elola, N. y Toransos, L.

2000. Evaluación educativa: una aproximación conceptual. Buenos Aires.

Escalante, E.

2005. Una perspectiva Ontológica de la Gestión Académica Universitaria. Argentina: Universidad Tecnológica Nacional.

Etkin, J.

2000. Política, gobierno y gerencia de las organizaciones. Argentina: Pearson Education.

2011. Gestión de la complejidad en las organizaciones. La estrategia frente a lo imprevisto y lo impensado. Argentina: Granica.

Flavell, J. H.

1971. First's disscusants comments. What is memory development the devolopment of Human Development. 14, 272-278. Citado por Fredy González (1996).

Gonzalez, F.

1996. Acerca de la Metacognición. Recuperado de: http://www.revistaparadigma.org.ve/Doc/ Paradigma96/doc5.htm

González, J., Galindo, N., Galindo, J. y Gold, M. 2004. Los paradigmas de la calidad educativa: de la autoevaluación a la acreditación. México: Unión de Universidades de América Latina.

Guerra, J.

2003. Metacognición: Definición y enfoques teóricos que la explican. Revista Electrónica de Psicología Iztacala. Vol. 6. No. 2. Universidad
Autónoma de México. Recuperado de http:// www.iztacala.unam.mx/carreras/psicologia/ psiclin/vol6num2/Metacognicion.html

Hernández, R. et. al.

2006. Metodología de la investigación. 4ta. Edición. México: McGraw-Hill.

Hunt, T.

1997. Desarrolla tu capacidad de aprender. La respuesta a los desafíos de la Era de la Información. Barcelona. Urano, citado por Antonio Ontoria (2003).

Inche, J., Chung, A. y Salas, J.

2010. Diseño e implementación de un sistema de gestión de calidad académico administrativa en la Unidad de Posgrado de la Facultad de Ingeniería Industrial de la UNMSM. Revista de la Facultad de Ingeniería Industrial 13 (2). 09-14. Perú: UNMSM.

\section{INNCREA CHILE}

2007. Desarrollando nuevas habilidades docentes, generando impacto. Quito. Citado por Nelson Raza, 2008. Texto de Estudio del Módulo de Gerencia de Centros de Educación Superior. ESPE.

Kauffman, K.

1998. Principios de la Calidad Total. Citado por Nelson Raza, 2008. Texto de Estudio del Módulo de Gerencia de Centros de Educación Superior. ESPE.

Kotter, J.

1990. El factor liderazgo. Madrid. Ed. Díaz de Santos, citado por Juan Manuel Manes, 2011. Gestión estratégica para instituciones educativas. Colección Cuadernos. 3ra., reimpresión. Argentina: Granica.

Lamata, R. \& Domínguez, R. (Coord.).

2003. La construcción de procesos formativos en educación no formal. Madrid: Narcea.

López, S.

2011. Sistemas de calidad. Implantación de diferentes sistemas en la organización. Colombia: Ideaspropias. 
Mesta, R.

2006. ¿Qué es la transparencia? Recuperado de: http://boards5.melodysoft.com/transparencia/que-es-la-transparencia-4.html

\section{METACOGNICIÓN}

2012. Recuperado de: http://pedablogia.wordpress.com/2007/03/16/la-metacognicionuna-estrategia-necesaria/

Nolker, H. y Schoenfeldt, E.

1983. Formación profesional: Enseñanza, currículo, evaluación. España. Editorial Reverté.

Ontoria, A.

2003. Potenciar la capacidad de aprender a aprender. Colección para educadores. Tomo 1. México. Alfaomega editores.

Owlia, M.

2010. A framework for quality dimensions of knowledge management systems, Total Quality Management \& Business Excellence, 21: 11, 1215-1228. Recuperado de: http://dx.doi.org /10.1080/14783363.2010.529351

Pacheco, L.

2000. La Gestión Académica Universitaria. Documento. Quito.

Porret, M.

2010. Gestión de personas. Manual para la gestión del capital humano en las organizaciones. 4ta. Edición. Madrid: ESIC.

Pulgar, J.

2005. Evaluación del aprendizaje en educación no formal. Recursos prácticos para el profesorado. Madrid: Narcea.

Raza, N.

2008. Cultura de la calidad total en la Educación. Texto de estudio de la Maestría en Docencia Universitaria. Sangolquí: ESPE.
Rincón, S.

2008. "Perspectivas gerenciales en el siglo XXI: Modelos, alcances y retos". Revista Venezolana de Gerencia (online). Vol. 13. No. 42, 179-180. Recuperado de: http:// www.scielo.org.ve/scielo.php?pid=S131599842008000200001\&script=sci_arttext

Rojas, J.

2006. Gestión Educativa en la sociedad del conocimiento. Bogotá: Cooperativa Editorial Magisterio.

Rosales, C.

2000. Evaluar es reflexionar sobre la enseñan$z a$. Madrid: Narcea.

Rosenblatt, Y.

2012. Metacognición. Recuperado de: http:// www.psicopedagogia.com/definicion/metacognicion

Zamboni, L.y Gorgone, H.

s. f. "Propuestas innovadoras en la gestión académica”. III Coloquio internacional sobre gestión universitaria en América del Sur. Recuperado de: http://rapes.unsl.edu.ar/Congresos_realizados/Congresos/III\%20Encuentro/ Completos/ZAMBONI.pdf

Zarate, J.

2008. Gestionar en equipo. Preguntas clave. Madrid: ESIC.

Zenteno, $\mathrm{P}$.

2012. Metacognición. Recuperado de: http:// www.psicopedagogia.com/definicion/metacognicion 